\title{
Electrical Injuries and Neurosurgery: A Case Report and Review of Literature
}

\author{
Sumeet Narang ${ }^{1}$ Ganesh Kumar Manoharan ${ }^{2}$ Jaspreet Singh Dil ${ }^{1} \quad$ A Raja ${ }^{1}$ \\ ${ }^{1}$ National Neurosciences Mission, Adarsha Super-Specialty Hospital, \\ Manipal-Udupi, Karnataka, India \\ 2 Department of Neurosurgery, Velammal Medical College, Madurai, \\ Address for correspondence Sumeet Narang, MBBS, National \\ Neurosciences Mission, Adarsha Superspecialty Hospital, Manipal- \\ Tamil Nadu, India \\ Udupi 576101, Karnataka, India (e-mail: sumeet_nvs@hotmail.com). \\ Indian J Neurotrauma
}

\begin{abstract}
Introduction Electrical injuries account for 5 to $27 \%$ of admissions to burn units. The nervous system is affected in as much as $21 \%$ of nervous injuries, with reported mortality.

Case Report The authors report a case of a patient presenting to the neurosurgical

Keywords

- burns

- electrical

- injuries

- neurosurgery

- TBI service with a traumatic brain injury (TBI) caused due to an electrical burn. Available data was reviewed through a PubMed search of literature, with special attention to the nature of presentation, classification of such injuries, the pathophysiology of the events that arise, complications to be expected, and the guidelines for management. Conclusion It is possible for electrical injuries to cause TBIs requiring neurosurgical intervention.
\end{abstract}

\section{Introduction}

While burns account for 20 to $40 \%$ of hospital admissions, 5 to $27 \%$ of those are attributed to electrical burn injuries. ${ }^{1}$ Although it is evident that burns are common causes of morbidity and mortality in the world, it is relatively uncommon for the neurosurgeon to be the doctor attending to a burn victim; yet it is seldom up to anyone else to deal with the unfortunate perils that affect the human brain, regardless of the cause.

The authors reported one such case of a patient presenting to the neurosurgical service with a traumatic brain injury (TBI) caused due to an electrical burn.

\section{Objectives}

To highlight the possibility of electrical burns causing TBI requiring immediate neurosurgical intervention and the association between the two entities.

DOI https://doi.org/ 10.1055/s-0041-1739481. ISSN 0973-0508.
To review the available data on such injuries, with special attention to the nature of presentation, the pathophysiology of the events that arise, complications to be expected, and the guidelines for management.

\section{Case Report}

History: A 35 year old male manual laborer from coastal Karnataka was brought to the emergency department with polytrauma sustained due to alleged history of electrocution, followed by fall from height while working on an electrical tower.

Physical examination: On initial assessment, the patient had a pulse rate (PR) of 64 /minute, blood pressure (BP) of $90 / 60 \mathrm{~mm}$ of $\mathrm{Hg}$, respiratory rate (RR) of 10/minute, unconscious with Glasgow coma scale (GCS) score of 4/15 (E1V1M2) with decerebration noted bilaterally, and pupils bilaterally fixed at $3 \mathrm{~mm}$, nonreacting to light. An emergency electrocardiogram (ECG) indicated atrial fibrillation. (c) 2021. Neurotrauma Society of India. All rights reserved.

This is an open access article published by Thieme under the terms of the Creative Commons Attribution-NonDerivative-NonCommercial-License, permitting copying and reproduction so long as the original work is given appropriate credit. Contents may not be used for commercial purposes, or adapted, remixed, transformed or built upon. (https://creativecommons.org/ licenses/by-nc-nd/4.0/)

Thieme Medical and Scientific Publishers Pvt. Ltd., A-12, 2nd Floor, Sector 2, Noida-201301 UP, India 


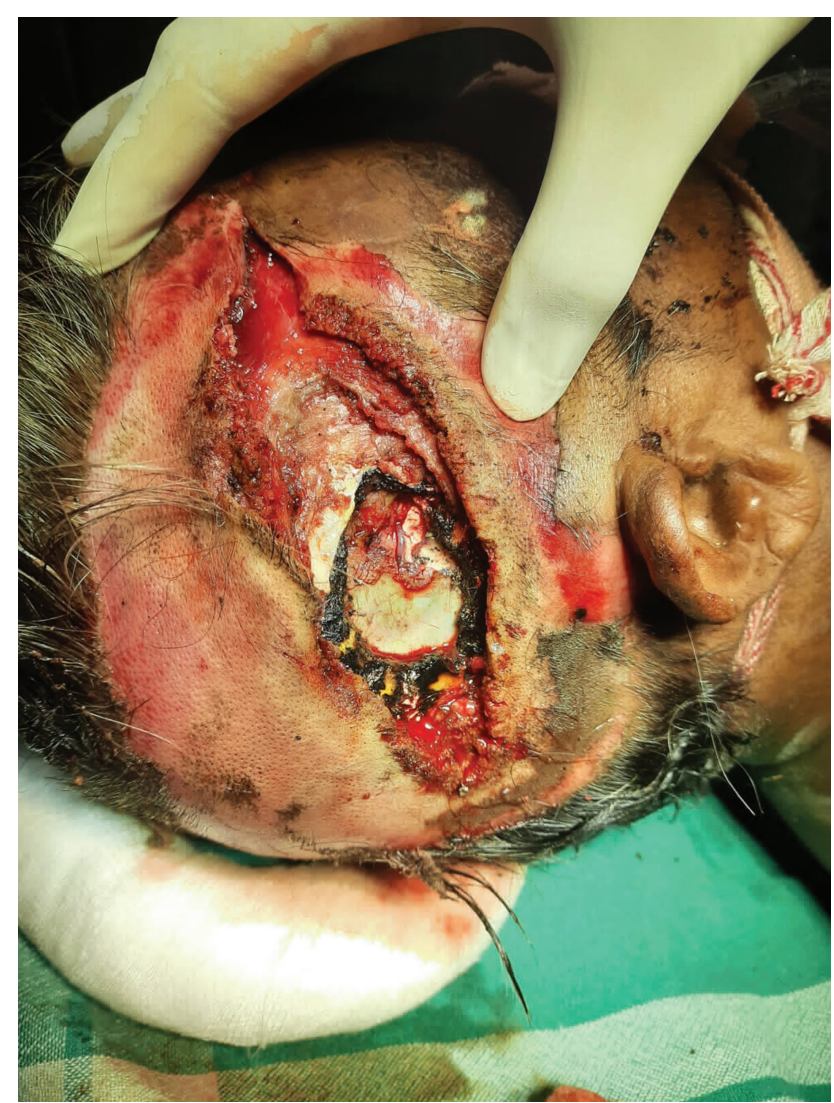

Fig. 1 Entry wound at the time of presentation (as described).

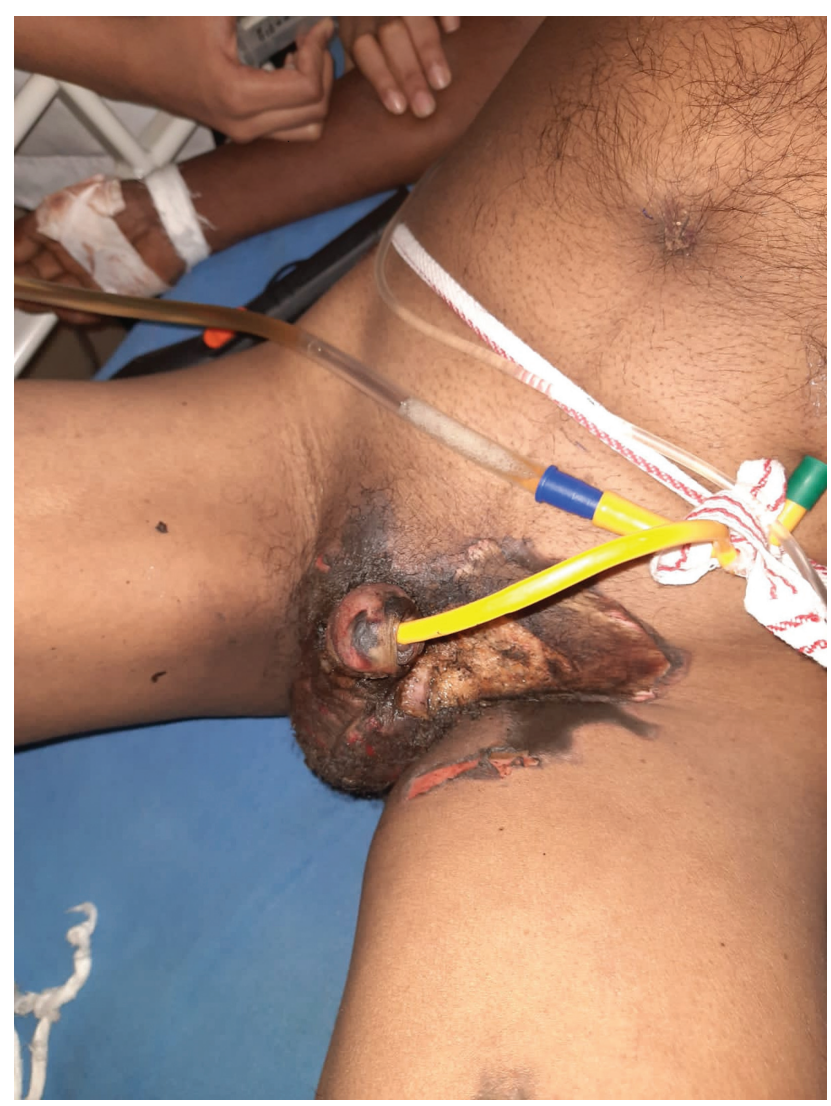

Fig. 2 Exit wound at the time of presentation (as described).
Further physical examination revealed the following:

- A $25 \times 5 \mathrm{~cm}$ laceration over the right parietal region of the scalp with blackish charred edges surrounded by hairless and hyperemic skin, with underlying open skull fracture surrounded by haematoma, and with a circular calvarial defect measuring approximately $7 \mathrm{~cm}$ in diameter, beneath which was visible the underlying dura seen to be ruptured with irregular margins along with hernia cerebri, that is, extruding gray matter: suggestive of both a possible entry point for electrical current as well as the result of blunt trauma as the underlying mechanism of the injury (-Fig. 1).

- Black charred skin over the pubis and scrotum, with superficial burns over the external genitalia and surrounding regions of the pubis and perineum, no evident signs of urethral injury: suggestive of a possible exit point for electrical current (-Fig. 2).

- Total external body surface area burnt as per the Lund and Browder chart $^{2}:<10 \%$

- No visible bony deformities of the long bones of the limbs and negative pelvic compression test.

Management: The patient was immediately intubated with a 7.5-mm endotracheal tube and ventilated, cannulated with wide bore intravenous (IV) cannulas in both forearms, subclavian IV access established, and fluid resuscitation initiated with Ringer Lactate, catheterized with a 16F Foley catheter, and transferred for an emergency computed tomography (CT) scan of the head, chest, and abdomen.

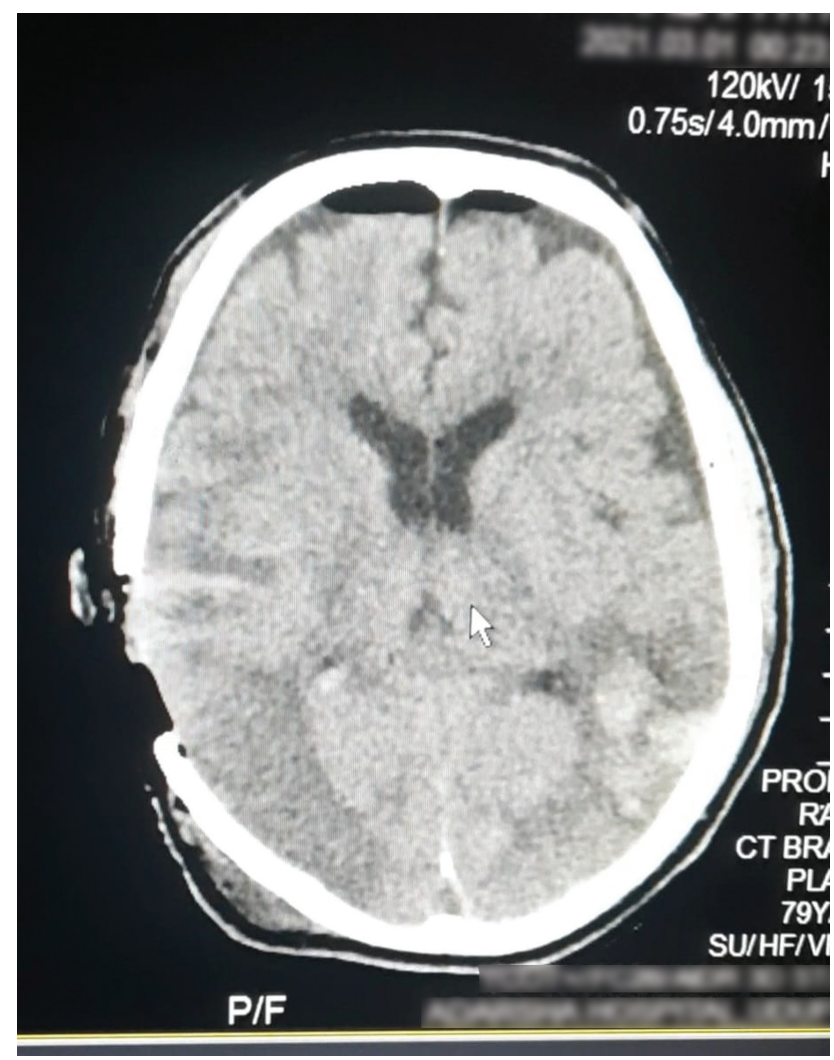

Fig. 3 CT-Scan at admission. 


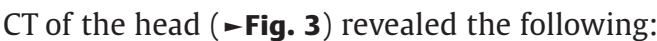

- Right parietal scalp haematoma.

- Open right parietal skull fracture.

- Bifrontal pneumocephalus and subdural hygroma.

- Right parietal contusions and corresponding contrecoup left parietal contusions.

- Traumatic subarachnoid hemorrhage.

- Cerebral edema, no transfalcine herniation.

The patient was immediately transferred to the operating theater in view of clinical, radiological, and emergent indications, while correction of hematological and cardiac abnormalities was initiated. The wound was thoroughly debrided-necrosed skin margins were excised and underlying skull wound washed with saline, with least possible contact to the ruptured dura and visible brain, which appeared contused with engorged veins. The subdural space was packed with hemostatic gelfoam, and dura was left open, following which the skin was approximated in a single layer with 2-0 non-absorbable nylon sutures (-Fig. 4). The wound was bandaged and dressed with adequate padding over the site of injury.

Patient's perineum was also carefully debrided and dressed adequately, and fluid resuscitation continued along with correction of hyperkalemia before patient was transferred to the intensive care unit for monitoring. Postoperatively, patient had PR 68/minute, BP 100/60, was on mechanical ventilation, and was draining adequate urine. He was managed with IV anticonvulsants, diuretics, antiarrhythmics

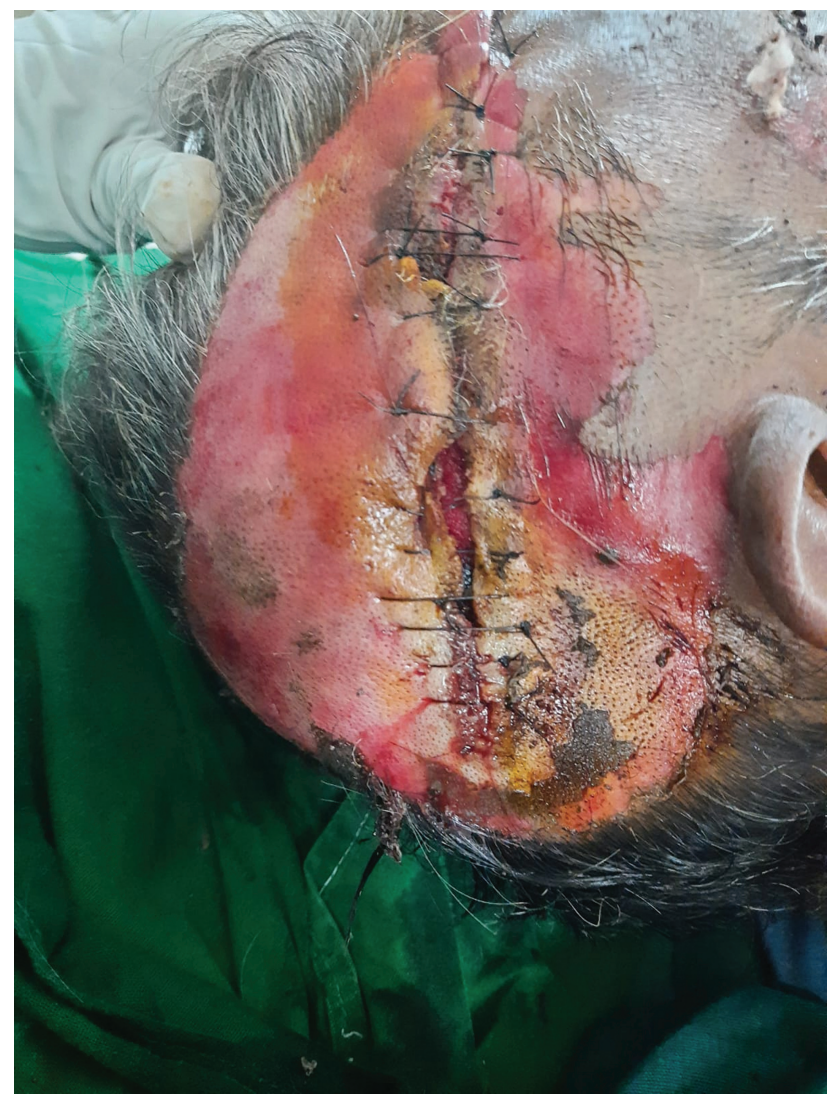

Fig. 4 Post-operative wound.
The patient, who had been brought in the late hours of the evening, had physiologically survived the night with his vital signs holding steady as was postop, with no improvement in GCS. Unfortunately, he was discharged against medical advice the following morning, owing to circumstances that are fairly or unfairly attributed to human beings.

\section{Discussion}

Electrical energy, apart from being the stuff of legends, natural and cosmic events, and popular abstract connotations, interestingly also plays many physiological roles in the human body. As students of the nervous system, we are aware that it is electrical energy that forms the basis of the intricacies of the system, ranging from the membrane potential to every action potential to the results of those minilightning bolts that also include the rhythms of the cardia.

One can endlessly list the myriad contributions of electricity to the life of the human being, and the neurosciences, whether it be the electric chair used for criminal executions in the yesteryears, developed by a person from the medical community, electroconvulsive therapy (ECT) used to treat psychiatric disorders like severe depression, or investigative roles of electroencephalogram/cardiogram/myograms (EEG/ECG/ EMG) to pacemakers and deep-brain stimulation.

It is however the bane of the same energy that could burn, causing devastating and life-threatening injuries. Studies from different parts of the world have shown that electrical burn injuries account for as much as 5\% of admissions to burn units. Electrical burns are responsible for as many as 1000 deaths per year in the United States. More than $50 \%$ of all electrical burn injuries are nonintentional. While males have seen to be more affected overall, the cause usually being occupational, electrical burns in the household setting are the cause of such injuries among women and children. Individuals in the age group of 18 to 40 years are more frequently affected. ${ }^{1,3,4}$

The major determinant for the severity of the injury is the voltage of the current that passes through the victim's body, and the nature of the current, that is, direct current (DC) or alternating current (AC). For this reason, electrical burns are broadly classified as follows:

(i) High-voltage burns: where the voltage is $1000 \mathrm{~V}$ or greater. These are more common, accounting for $57.71 \%$ of electrical burns. High-voltage burns may be "true high-voltage burns" or there is also an entity called "flash burns," where an arc of highvoltage current emits heat that causes superficial burns, commonly to the hand and face. ${ }^{1,5}$,

(ii) Low-voltage burns: where the voltage is less than $1000 \mathrm{~V}$, as seen in domestic burns, accounting for $42.29 \%$ of electrical burns. ${ }^{1,5}$

High-voltage injuries cause more extensive tissue damage and loss, and may involve more systemic complications like fatal arrhythmias and renal failure or even death, when 
compared with low-voltage burns. The standard classification of the degree of severity of the burn injury, regardless of the cause, is based on the thickness or depth of the tissue involved in the form of superficial (first degree), partial thickness (second and third degree burns), and full thickness (fourth degree burns). ${ }^{1}$

\section{Pathophysiology}

What makes electrical burns interesting is the mechanism by which it acts. Any electrical source must have an entry point through which the current enters the body: usually the head or the hands. The current traverses through the body along the path of least resistance before it leaves the body through an exit point, which is usually the feet. While this is the general principle, it may not apply to an alternating source of current where the current travels within the body. 1,6,7

Apart from the voltage and the nature of the current, it is the duration of exposure and the path taken by the current in the body that determines the extent of the internal injuries, and this is where the conversation can be directed toward the nervous system, which is affected in as much as $21 \%$ of cases. The nerves of the body, the large ones being myelinated, and all being natural conductors of the electricity, offer low resistance, similar to blood vessels, when compared with skin, muscle and bone, which offer high resistance. As a result, the nerves and blood vessels favor the path of the current, and this is responsible for the neurological and the cardiac sequelae; while muscles and other soft tissue may suffer the direct impact of the thermal injury generated. ${ }^{1,5-8}$

Studies have shown that as the electrical current travels through the body, the neurological effects are caused due to the following:

- Electroporation: refers to the opening of pores in the cell membrane, initiating a cascade of events that favor cellular death.

- Vascular injury: due to spasm and release of vascular inflammatory mediators, leading to neuronal ischemia, neuronal chromatolysis, and microglial activation.

- Demyelination: due to alteration of the nature of the proteins and lipids.

- Release of thermal injury, causing destruction of macromolecules.

- Neurohumoral hypothesis: suggesting that electricity aids in circulating substances, specifically cortisol, a stress hormone, and generation of free radicals acting at a distance and by hyper-stimulation of glutamate receptors. The cointeraction of all of these may also be responsible for the delayed effects in the central nervous system (CNS) as well as peripheral nervous system (PNS).

Following the injury, due to the above mechanisms, 1,6,9,10 events occur that have effects at different times, which can be categorized $^{1,11}$ as follows:

- Immediate and transient, for example, amnesia and loss of consciousness.

- Immediate and prolonged, for example, hemorrhage or infarction.
Neurological injuries caused by electrocution.

\begin{tabular}{lll}
\hline Type of nervous system & Immediate & Delayed \\
\hline Central nervous system & Confusion & Transverse myelitis \\
& Loss of consciousness & Amyotrophic lateral sclerosis \\
& Amnesia & Ascending paralysis \\
& Acute stroke & Personality changes \\
& Seizure & Delayed brain atrophy \\
& Headache & \\
& Focal brain necrosis \\
& Transection of spinal cord \\
& Rupture of aneurysm & \\
\hline \multirow{2}{*}{ Peripheral nervous system } & Nerve palsy & Neuropathies \\
& & \\
Autonomic nervous system & Raynaud phenomenon & Complex regional pain syndrome \\
& Horner's syndrome & \\
& Keraunoparalysis &
\end{tabular}

Fig. 5 Effects of electrical injuries to the different divisions of the nervous system.

- Delayed and progressive, for example, demyelination.

- Secondary or indirect effects, for example, TBI.

The effects may also be categorized based on the various divisions of the nervous system affected, as presented below ${ }^{1}$ and summarized in - Fig. $\mathbf{5} \mathbf{- ~}^{12}$ :

\section{- Central Nervous System (CNS)}

(i) TBI: possibly a direct or an indirect effect, as seen in the case above, where the injury could have been caused due to the current entering the body, or the patient being thrown off balance after the electrocution and sustaining a head injury, or a combination of both. The injury may be a closed head injury or a penetrating injury and usually requires immediate surgical intervention.

One study has reported that as much as 35\% of electrical burn victims evaluated suffered head injuries, and 9.9\% of patients required neurosurgical treatment, including cranioplasty for calvarial injuries. ${ }^{4}$

(ii) Cerebral edema: secondary to electrocution affecting the brain matter itself and also as part of the cycle of the other listed complications.

(iii) Ischemia (stroke): as a consequence of vasospasm. Strokes following electrocution have been reported in specific individual instances as well. ${ }^{12}$

(iv) Seizures: due to electrical stimulation, occurring both as immediate and delayed consequence.

(v) Cerebellar syndromes: due to electrocution and subsequent ischemia or hemorrhage.

(vi) Hydrocephalus requiring shunt insertion.

(vii) IV thrombosis: also known to be related to seizures itself.

(viii) Myelopathy and spinal cord transection due to the course of the current, and a possibility of a BrownSequard-like presentation, with a combination of upper motor and lower motor neuron signs. 
(ix) Amyotrophic lateral sclerosis (ALS): delayed sequelae.

(x) Movement disorders: delayed sequelae.

- Peripheral Nervous System (PNS)

(i) Mononeuropathy commonly affecting, but not limited to, the nerves of the upper limb, as they are usual entry points. Median and ulnar neuropathies have been commonly reported. They could also be secondary to fracture of the long bones.

(ii) Polyneuropathy

(iii) Polyradiculopathy where there are multiple entry points.

(iv) Cranial nerve dysfunction where the head and its orifices are entry points. Patients have been found to have sensorineural hearing loss and associated symptoms like vertigo and tinnitus, apart from presentations attributed to the damage of individual nerves or a combination of more than one.

- Autonomic Nervous System (ANS)

(i) Autonomic cardiovascular complications are wellknown and cannot be ignored. Arrhythmias are a direct result of electrical injuries, and intensive and sequential cardiac monitoring is indicated in all electrical burn victims. ${ }^{13}$

(ii) Horner syndrome.

(iii) Bladder dysfunctions.

(iv) Sexual dysfunctions.

(v) Complex regional pain syndrome.

(vi) Reflex sympathetic dystrophy.

(vii) Keraunoparalysis is also known as Charcot's paralysis where the patient has temporary limb paralysis, pallor and pulselessness following a surge of catecholamine release.

It is for the management of the above-listed sequelae and complications that the neurosurgeon has a key role to play in the immediate and future care of the electrical burn victim alongside a multidisciplinary team to treat the individual as a whole, which we subsequently discuss.

- Other systemic complications

(i) Arrhythmia: Atrial and ventricular fibrillation are common and ST changes in the ECG have also been reported. It is also essential to monitor cardiacenzymes. ${ }^{13}$

(ii) Rhabdomyolysis and myoglobinuria with raised creatine phosphokinase levels, further leading to:

(iii) Acute renal failure.

(iv) Electrolyte abnormalities, with special attention to potassium levels that have definite association to the cardiac rhythm abnormalities as a result of the thermal injuries and fluid loss that requires rigorous resuscitation.

(v) Spinal injuries and fractures or dislocation of long bones and also of the commonly encountered thoracic and lumbar spine.

(vi) Ocular injuries and their sequelae-like cataract and keratoconjunctivitis. (vii) Abdominal visceral injuries have been widely documented and includes a wide spectrum from mild injuries requiring conservative management as well as severe cases where laparotomy and exploration is doubtlessly indicated and where mortality has been significant. ${ }^{6}$

(viii) Sepsis and subsequent multiorgan dysfunction syndrome (MODS).

\section{Management}

Management of any burn victim, electrical or not, is to be rigorous from the time the patient is wheeled into the emergency room. All possible injuries, immediate as well as delayed, must be imagined from the initial assessment itself. Following emergency management (airway breathing and circulation $[A B C]$ ), the patient must receive fluid resuscitation as per the formulae adopted by the center, the Parkland formula still being the standard. The patient must undergo thorough and regular investigations, the most important of which is the ECG, before bloodwork including cardiac enzymes and creatine phosphokinase (CPK) levels, and CT scan of the head and neck, thorax and abdomen, to rule out the extent of potential internal injuries. ${ }^{1,4,6,13}$

For the neurosurgeon, the authors reiterate the need for a comprehensive neurological assessment and evaluation for the need for surgery. Open head injuries must be managed acutely with the high-risk of infection in mind, and all other pathologies must be urgently excluded, regardless of the condition of the pupils that may be misleading as a result of autonomic dysfunction, as described. Immobilization of the spine must never be overlooked. ${ }^{1,6}$ Measures must be taken to ensure that morbidity and mortality arising, due to a neurosurgical pathology, is avoided at all costs.

Burn victims inevitably require to undergo multiple plastic surgical interventions like debridement, skin grafting, chronic wound dressing, contracture releases, etc. apart from the constant medical treatment needed, making them chronic patients, bearing all the burden that comes with it, such as the complications of being bedridden, who later also require intensive rehabilitation. 1,6

The most important step in the management of burns remains the prevention of burns, and the doctor plays a tremendous role in educating the public about the causes, effects, and management of burns.

\section{Conclusion}

It is possible for electrical injuries to have direct or indirect but certainly far-reaching effects on the nervous system; hence, awareness on the subject and appropriate approach is critical for the neurosurgeon/neurologist in saving the life of the patient.

\section{Note}

This article was presented (Award Paper) at the 29th Annual Conference of the Neurotrauma Society of India (NTSI), Madurai, on August 28, 2021. 
Electrical Injuries and Neurosurgery Narang et al.

\section{Funding}

None.

\section{Conflict of Interest \\ None declared.}

\section{References}

1 Yiannopoulou KG, Papagiannis GI, Triantafyllou AI, et al Neurological and neurourological complications of electrical injuries. Neurol Neurochir Pol 2021;55(01):12-23

2 Lund CC, Browder NC. The estimation of areas of burns. Surg Gynecol Obstet 1944;79:352-358

3 Gentges J, Schieche C. Electrical injuries in the emergency department: an evidence-based review. Emerg Med Pract 2018; 20(11):1-20

4 Thomas J, Sreekumar NC, Shankar C, James A. Complications and outcome of electrical burns in Manipal, India: 6-year institutional report. World J Plast Surg 2020;9(01):14-21

5 Hettiaratchy S, Dziewulski P. ABC of burns: pathophysiology and types of burns. BMJ 2004;32874531427-1429
6 Marques EG, Júnior GA, Neto BF, et al. Visceral injury in electrical shock trauma: proposed guideline for the management of abdominal electrocution and literature review. Int J Burns Trauma 2014;4(01):1-6

7 Friedstat J, Brown DA, Levi B. Chemical, electrical, and radiation injuries. Clin Plast Surg 2017;44(03):657-669

8 Dechent D, Emonds T, Stunder D, Schmiedchen K, Kraus T, Driessen S. Direct current electrical injuries: A systematic review of case reports and case series. Burns 2020;46(02):267-278

9 Andrews CJ, Reisner AD. Neurological and neuropsychological consequences of electrical and lightning shock: review and theories of causation. Neural Regen Res 2017;12(05):677-686

10 Reisner AD. Possible mechanisms for delayed neurological damage in lightning and electrical injury. Brain Inj 2013;27(05):565-569

11 Cherington M. Central nervous system complications of lightning and electrical injuries. Semin Neurol 1995;15(03):233-240

12 Kokatnur L, Rudrappa M. Acute stroke due to electrocution: uncommon or unrecognized? Case Rep Neurol Med 2016; 2016:9510863

13 Arnoldo B, Klein M, Gibran NS. Practice guidelines for the management of electrical injuries. J Burn Care Res 2006;27(04): 439-447 